\title{
FORMULATION AND CHARACTERIZATION OF GELUCIRE PELLETS FOR SUSTAINED RELEASE OF IBUPROFEN
}

\author{
Gihan Nabil Fetih
}

Deptartment of Pharmaceutics, Faculty of Pharmacy, Assiut University, Assiut, Egypt

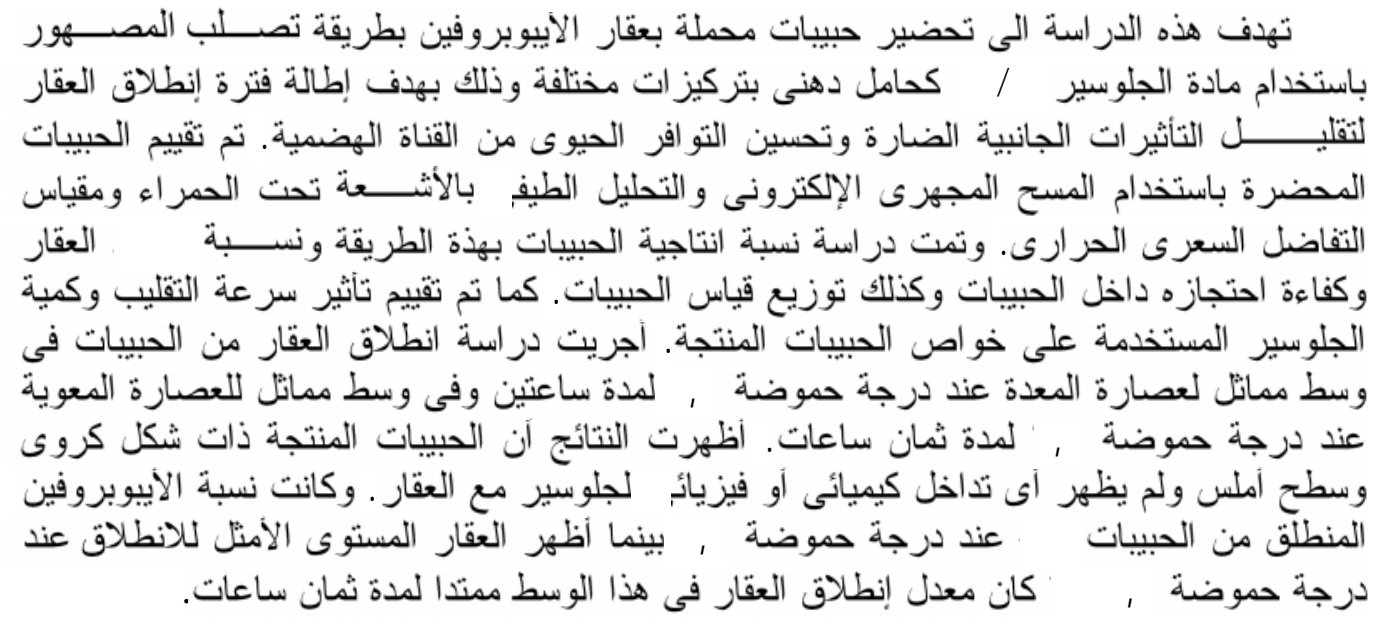

The aim of the present study was to develop ibuprofen (IBU) - loaded pellets by melt solidification technique using Gelucire 50/13 (GL) as a lipid carrier in different concentrations. This system was intended to prolong the drug release in order to minimize the drug related adverse effects and improve bioavailability in different gastrointestinal tract conditions. The prepared pellets were evaluated using scanning electron microscopy (SEM), Infrared spectroscopy (IR), and Differential scanning calorimetry (DSC) studies. Process yield, drug loading, encapsulation efficiency, and particle size distribution were also investigated. The effect of agitation speed and amount of GL on pellets properties was evaluated. In-vitro drug release of ibuprofen from prepared pellets was studied in $\mathrm{HCl}$ buffer (pH1.2) for $2 \mathrm{hrs,} \mathrm{and} \mathrm{in}$ phosphate buffer ( $p H$ 7.4) for up to 8 hrs. The obtained pellets were spherical in shape with smooth surfaces; and GL showed no interaction with the drug. The release of drug from the pellets showed low percentage of drug release in $\mathrm{pH}$ 1.2. However, at $\mathrm{pH} 7.4$ the obtained results showed that optimum levels of drug were released in a sustained manner.

\section{INTRODUCTION}

Pharmaceutical formulation techniques, which do not involve using organic solvents, are preferred due to stringent global requirements of quality. Hence, many reports are published on techniques such as melt granulation $^{1 \& 2}$, melt extrusion ${ }^{3 \& 4}$, melt dispersion $^{5-7}$, and melt solidification ${ }^{8-10}$ for controlled drug delivery systems formulation.

Lipids and waxes are considered as good alternatives to polymers in the design of controlled drug delivery systems due to their advantages like low melt viscosity, thereby obviating the need of organic solvents for solubilization, the absence of toxic impurities such as residual monomers catalyst and initiators, their potential biocompatibility and biodegradability and prevention of gastric irritation by forming a coat around the gastric irritating drug ${ }^{11 \& 12}$.

Among waxy materials, Gelucires are a family of relatively inexpensive materials, comprising mixtures of mono-, di-, and triglycerides and also poly (ethylene glycol) esters of fatty acids. They are available with a range of properties depending on their hydrophilic lipophilic balance (HLB; 1-18) and melting point $\left(33-65^{\circ} \mathrm{C}\right)$ range ${ }^{13-15}$. Gelucires containing only polyethylene glycol 
(PEG) esters (Gelucire 55/18) are generally used in the preparation of fast-release formulations, while those containing only glycerides or a mixture of glycerides and PEG esters (Gelucire 54/02, 50/13, 43/01) are used in the preparation of sustained-release formulations ${ }^{16 \& 17}$

The presence of both hydrophobic glycerides and more hydrophilic PEG esters results in a wide range of hydrophobicity and drug release rates. This versatility makes their use very promising as base materials for the production of sustained-release formulations ${ }^{18}$.

A number of design options are available for the preparation of controlled release formulations to modify oral absorption by matrix pellets ${ }^{19}$. Pellets are receiving increased attention. They are defined as spherical, freeflowing granules with a narrow size distribution, typically varying between 500 and $1500 \mu \mathrm{m}$ for pharmaceutical application $\mathrm{s}^{20}$. The interest in pellets as dosage forms (filled into hard gelatin capsules or compressed into disintegrating tablets) has been increasing continuously, since their multiparticulate nature offers some important pharmacological as well as technological advantages over conventional single-unit solid dosage forms. Smaller sized pellets are rapidly emptied from the stomach regardless of the feeding state of the patient and influence the gastric emptying rate on the upper gastro-intestinal transit time ${ }^{20}$. Multi particulate unit dosage forms such as pellets, reduces the intra and inter-subject variability in gastric emptying times and exhibits easy distribution of the contents at gastrointestinal tract, compared to single-unit dosage forms ${ }^{20}$. The uniform dispersion of a drug into pellets reduces the risk of high drug concentration, dose dumping and irritating effect on gastric mucosa. Furthermore, drug absorption is maximized and peak plasma fluctuations are reduced. Spherical shape of the pellets would lead to good flow properties which ensure narrow size distribution and good content uniformity ${ }^{21}$.

Ibuprofen (IBU),-methyl-4-(2-methylpropyl)-benzene acetic acid is a non-steroidal anti-inflammatory drug used to treat rheumatoid arthritis, osteoarthritis and mild to moderate pain. The gastrointestinal irritation and ulcerogenic effect along with short half-life $(1.8-2.0 \mathrm{hrs})$ has lead to the design of sustained release formulations of $\mathrm{IBU}^{22-24}$. Due to its low melting point and hydrophobic nature many attempts have been made to develop wax based sustained release formulations ${ }^{5,6 \& 25}$.

Melt solidification/melt dispersion technique basically involves emulsification of the molten mass in the aqueous phase followed by its solidification by chilling. Melt dispersion technique reported by Adeyeye et al. ${ }^{5}$ and Bodmeier et l. $^{24}$ for the development of IBU microspheres involves emulsification of IBU-wax melt at temperature greater than the melting point of IBU followed by cooling to room temperature. In this technique, the drug: wax ratios were significantly high from 1:1 to 1:4. The microspheres obtained have drug loading in the range of $15-40 \%$, with particle size between 50-300 m.

IBU melt has low viscosity and ability to remain in the liquid state for sufficiently longer period of time even at low temperatures, it has a very low glass transition temperature $\left(\mathrm{t}_{\mathrm{g}}\right)$ of $<$ $-30^{\circ} \mathrm{C}^{25}$. Solidification of melt can be accelerated by application of shear. In such cases emulsification can be carried out at temperature well below its melting point. On the basis of these properties a novel single step melt solidification technique (MST) was designed by Paradkar et $a .^{26}$, where emulsification and solidification of the melt in aqueous phase was carried out at $5^{\circ} \mathrm{C}$. For further retardation of drug release and improvement in sphericity, cetyl alcohol (CA) was incorporated. The inherent strength of the melt solidified bonds helps to reduce the amount of excipient required to retard the release $^{8}$. The maximum amount of wax that could be incorporated in low temperature MST depends on the properties of the drug; it was $12.5 \% \mathrm{w} / \mathrm{w}$ for $\mathrm{IBU}^{8}$ and $25 \% \mathrm{w} / \mathrm{w}$ for flurbiprofen ${ }^{9}$. However, this percentage is significantly low as compared to the melt dispersion technique involving high temperature emulsification.

In the present study, the objective was to prepare IBU- Gelucire 50/13 (IBU-GL) pellets by melt solidification technique as a sustained release formulation of IBU. The effect of GL concentration on the properties of the prepared pellets was evaluated. Scanning electron microscopy, IR spectroscopy, DSC, and invitro drug release were utilized to characterize the pellets. 


\section{EXPERIMENTAL}

\section{Materials}

Ibuprofen (IBU) was kindly supplied by Kahira Pharmaceutical \& Chemical Industries Co. (Cairo, Egypt), Gelucire 50/13 (GL) was obtained from Gattefosse' (Saint- Priest, Cedex, France), all other solvents and chemicals were of analytical grade.

\section{Methods}

\section{Preparation of IBU-GL pellets}

Pellets were prepared by meltsolidification method. A mixture of IBU (2 g) and GL $(200,250$, or $300 \mathrm{mg})$ was melted and stirred on a water bath maintained at $80^{\circ} \mathrm{C}$ to form a uniform molten mass. The IBU-GL melt was poured in $100 \mathrm{ml}$ water pre-cooled to $20^{\circ} \mathrm{C}$, and was stirred continuously (at 800 , 1000 , or 1200) rpm using constant speed stirrer with propeller blade (Eurostar power controlvisc, IKA Labortecnik, Germany). The IBUGL pellets obtained after solidification of dispersed droplets were separated by filtration, washed with distilled water and dried at room temperature for $24 \mathrm{hrs}$. The formulation composition and preparation conditions are summarized in Table 1.

Table 1: The process conditions for preparing of IBU-GL pellets.

\begin{tabular}{|c|c|c|c|}
\hline \hline Code & $\begin{array}{c}\text { IBU } \\
(\mathrm{g})\end{array}$ & $\begin{array}{c}\text { GL } \\
(\mathrm{mg})\end{array}$ & $\begin{array}{c}\text { Agitation speed } \\
(\mathrm{rpm})\end{array}$ \\
\hline F1 & 2 & 200 & 800 \\
\hline F2 & 2 & 200 & 1000 \\
\hline F3 & 2 & 200 & 1200 \\
\hline F4 & 2 & 250 & 800 \\
\hline F5 & 2 & 250 & 1000 \\
\hline F6 & 2 & 250 & 1200 \\
\hline F7 & 2 & 300 & 800 \\
\hline F8 & 2 & 300 & 1000 \\
\hline F9 & 2 & 300 & 1200 \\
\hline
\end{tabular}

\section{Evaluation of IBU-GL pellets}

\section{Yield, drug loading, and entrapment efficiency}

Pellets were weighed after drying and percent yield was calculated by using the following formula:

$\%$ yield $=$

$\left[\frac{\text { weightof pelletscollected }}{\text { weightof all componentsused for thepreparatio }}\right] \times 100$
For determination of drug content, $100 \mathrm{mg}$ pellets were triturated and dissolved in $100 \mathrm{ml}$ ethanol by sonication for $30 \mathrm{~min}$. The solution was analyzed spectrophotometrically at 222 $\mathrm{nm}^{8}$ (Shimadzu-50-02, Kyoto, Japan) after sufficient dilution with phosphate buffer $(\mathrm{pH}$ 7.4). Blank pellets were treated similarly. It was confirmed that the used excipient (GL) did not interfere with the spectrophotometric assay of the drug.

The percent drug loading $=$ $\left[\frac{\text { Amount of drug in the sampled pellets }}{\text { the weight of pellets }}\right] \times 100$

Percent entrapment was calculated by using the following formula:

$\%$ Drug entrapment $=$

$$
\left[\frac{\text { Calculated drug content }}{\text { Theoretical drug content }}\right] x 100
$$

\section{Particle size analysis}

Particle size distribution of the prepared pellets was studied by sieve analysis technique using a nest of standard sieves (USP).

\section{Scanning electron microscopy (SEM)}

The surface characteristics of the prepared pellets were examined with a scanning electron microscope (Joel, JSM-5400 LV, Japan) operated at an acceleration voltage of $15 \mathrm{kV}$. IBU-GL pellets were coated with gold palladium foil $(54 \mathrm{~nm})$ by sputter coater unit (SPI, sputter, USA) prior to examination.

\section{Infrared spectroscopy (IR)}

Samples (IBU, GL, and IBU-GL pellets) were mixed with $\mathrm{KBr}$ and compressed into disc using hydrolic pump (Shimadzu IR-470, Japan) under pressure of about 5 ton. The spectra were recoded over a range of $4000-300 \mathrm{~cm}^{-1}$.

\section{Differential scanning calorimetry (DSC)}

Thermograms of the samples (IBU, GL, and IBU-GL pellets) were obtained using differential scanning calorimetry (DSC-60, Shimadzu, Japan). Thermal analysis data were recorded using a TA 50I PC system with 
Shimadzu software programs. Indium standard was used to calibrate the DSC temperature and enthalpy scale. The samples (3-5 mg) were sealed in aluminum pans and heated at a constant rate of $10^{\circ} \mathrm{C} / \mathrm{min}$, over a temperature range of $25-200^{\circ} \mathrm{C}$. Inert atmosphere was maintained by purging nitrogen at rate of 30 $\mathrm{ml} / \mathrm{min}$.

\section{In-vitro drug release studies}

The release profiles of IBU from the prepared pellets were investigated in two different buffer solutions to mimic the various physiological gastrointestinal tract conditions. The $\mathrm{HCl}$ buffer of $\mathrm{pH} 1.2$ represented the gastric $\mathrm{pH}$ condition and phosphate buffer of $\mathrm{pH} 7.4$ simulated the intestinal condition. The dissolution process was carried out by using USP XXIII dissolution apparatus (Erweka DTD6, Duesseldorf, Germany). The drug loaded pellets (equivalent to $300 \mathrm{mg}$ of drug) were placed into the dissolution vessel containing $900 \mathrm{ml}$ of the release medium which was stirred at a constant speed of $100 \mathrm{rpm}$ and maintained at temperature $37 \pm 0.5^{\circ} \mathrm{C}$. The dissolution in $\mathrm{pH} 1.2$ medium was carried out for $2 \mathrm{hrs}$. and the dissolution in $\mathrm{pH} 7.4$ buffer solution was carried out for $8 \mathrm{hrs}$. At scheduled time intervals, the sample $(5 \mathrm{ml})$ was withdrawn and replaced with same volume of fresh dissolution medium. The withdrawn samples were filtered through a $0.45 \mathrm{~m}$ membrane filter and then evaluated for IBU concentration spectrophotometrically at 222 $\mathrm{nm}$ against a blank after appropriate dilution.

\section{RESULTS AND DISCUSSION}

This study aimed at using GL to prepare IBU pellets to retard the drug release using melt solidification technique. The melt solidification technique developed by Paradkar et al. $2003^{26}$ involved addition of molten IBUcetyl alcohol mass to a chilled aqueous phase maintained at $5^{\circ} \mathrm{C}$. During this process, emulsification and hardening was achieved within 2-3 min. This fast cooling and high shear caused formation of melt solidified bonds between the drug particles. Low process temperature minimized the drug loss. In this study GL was used as a waxy excipient in a higher concentration range (10-15\% w/w) and the IBU-GL molten mass was added to aqueous phase maintained at $20^{\circ} \mathrm{C}$, as $\mathrm{GL}$ could not remain in liquid state at $5^{\circ} \mathrm{C}$.

\section{Preliminary characterization of IBU-GL pellets}

The process yield of various prepared batches was in the range of $88.3-95.5 \%$. It was not affected by the amount of GL or agitation speed. Entrapment efficiency of various batches was in the range of 89.1-97.2\%. Drug loading and entrapment efficiency was found to be slightly higher for pellets prepared with lower amount of GL and slower agitation speed (these results are summarized in Table 2). Drug loading increased probably with increase in pellets size due to larger surface area, resulting in an increase in entrapment efficiency ${ }^{20}$. This could be confirmed by particle size analysis (discussed later). Maheshwari et al. $2003^{8}$ have reported that waxy flakes of cetyl alcohol (CA) were found to separate at concentrations higher than $12.5 \% \mathrm{w} / \mathrm{w}$ and also the sphericity was affected. This was not the case in this study, where the process yield was high and no flakes were separated up to $15 \% \mathrm{w} / \mathrm{w}$, and pellets were spherical but surface roughness was observed.

Table 2: Yield, drug loading, and entrapment efficiency of different IBU-GL pellet batches.

\begin{tabular}{|c|c|c|c|}
\hline Code & $\begin{array}{c}\text { Yield } \\
(\%)\end{array}$ & $\begin{array}{c}\text { Drug } \\
\text { loading (\%) }\end{array}$ & $\begin{array}{c}\text { Entrapment } \\
\text { efficiency }(\%)\end{array}$ \\
\hline F1 & $88.3 \pm 0.40$ & $88.2 \pm 0.21$ & $97.2 \pm 0.22$ \\
\hline F2 & $89.5 \pm 0.36$ & $87.0 \pm 0.34$ & $95.7 \pm 0.31$ \\
\hline F3 & $88.0 \pm 0.45$ & $85.9 \pm 0.22$ & $94.5 \pm 0.27$ \\
\hline F4 & $92.4 \pm 0.70$ & $82.8 \pm 0.35$ & $93.2 \pm 0.36$ \\
\hline F5 & $91.7 \pm 0.61$ & $81.8 \pm 0.51$ & $92.1 \pm 0.40$ \\
\hline F6 & $92.8 \pm 0.36$ & $81.2 \pm 0.23$ & $91.4 \pm 0.33$ \\
\hline F7 & $94.3 \pm 0.46$ & $78.4 \pm 0.20$ & $90.2 \pm 0.21$ \\
\hline F8 & $94.0 \pm 0.55$ & $78.0 \pm 0.26$ & $89.7 \pm 0.23$ \\
\hline F9 & $95.5 \pm 0.45$ & $77.5 \pm 0.42$ & $89.1 \pm 0.18$ \\
\hline
\end{tabular}

Mean values of 3 experiments \pm SD.

\section{Scanning electron microscopy (SEM) of the prepared pellets}

The SEM photomicrograph of the obtained IBU-GL pellets shows that the pellets were spherical with smooth surface as seen from (Fig. 1a). Pellets prepared with higher GL concentration $(15 \% \mathrm{w} / \mathrm{w})$ were also spherical but with rough surface (Fig. 1b). 

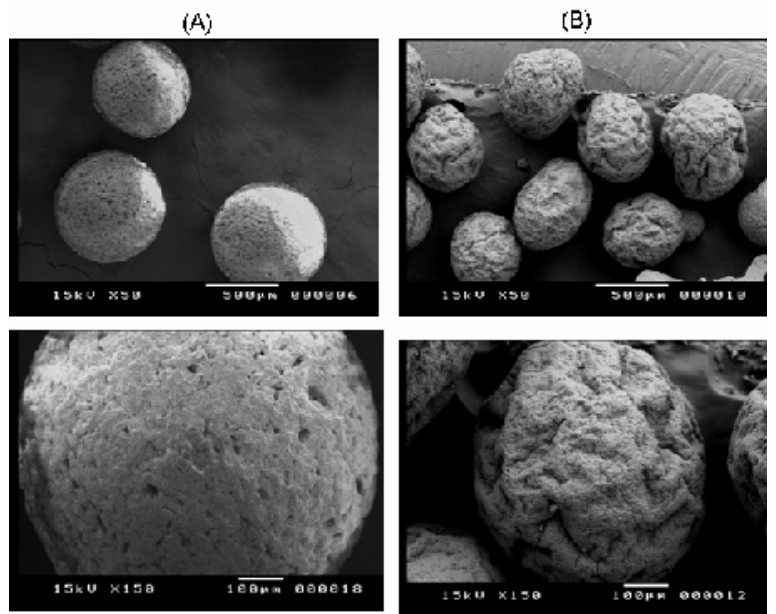

Fig. 1: SEM microphotographs of IBU-GL pellets. (A): Pellets of $10 \%$ w/w GL. (B): Pellets of $15 \%$ w/w GL.

\section{Infrared spectroscopy (IR) and Differential scanning calorimetry (DSC)}

The IR spectrum shows the characteristic peaks of ibuprofen at $1720 \mathrm{~cm}^{-1}$ and $2920 \mathrm{~cm}^{-1}$, due to carbonyl and hydroxyl stretching respectively (Fig. 2) indicating that the drug retains its identity in the formulation.

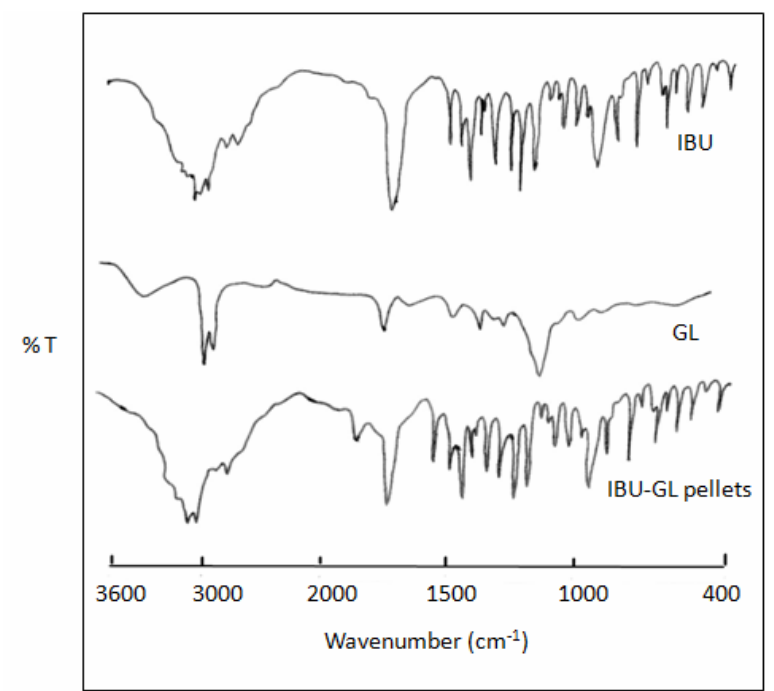

Fig. 2: IR spectra of IBU, Gelucire 50/13, and IBU-Gelucire pellets.

DSC thermogram of IBU-GL pellets (Fig. 3) shows two endotherms at 50 and $76.6^{\circ} \mathrm{C}$ due to the melting of GL and IBU respectively. No shift of drug peaks is observed indicating absence of any interaction between the drug and the excipient (GL).
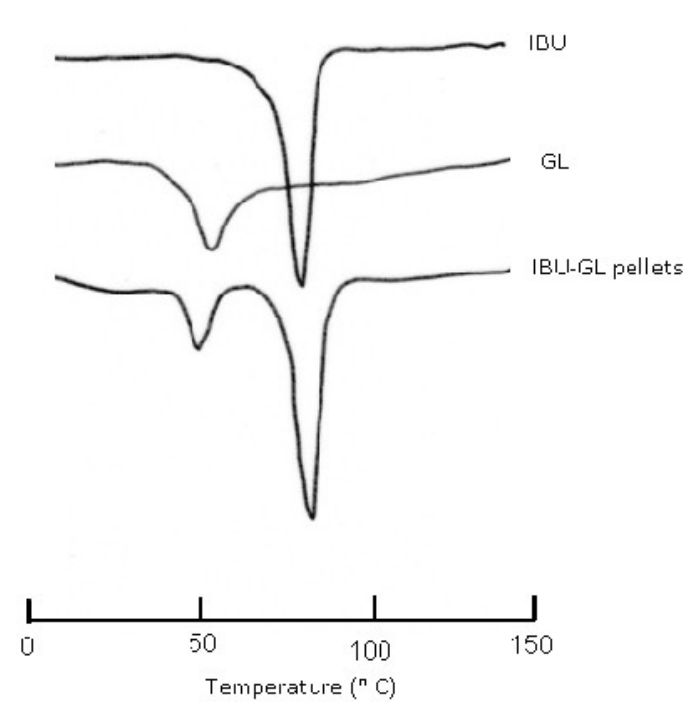

Fig. 3: DSC thermograms of IBU, Gelucire 50/13, and IBU-Gelucire pellets.

\section{Particle size analysis}

The particle size distribution for different prepared pellet batches is shown in figure 4 . The pellets of average size of 800-1200 $\mu \mathrm{m}$ are suitable for capsule filling ${ }^{8}$. Batches processed at lower GL concentrations and lower agitation speed favored larger particle size (Fig. 4), this explains higher drug loading and higher entrapment efficiencies for pellets in those batches as mentioned earlier.

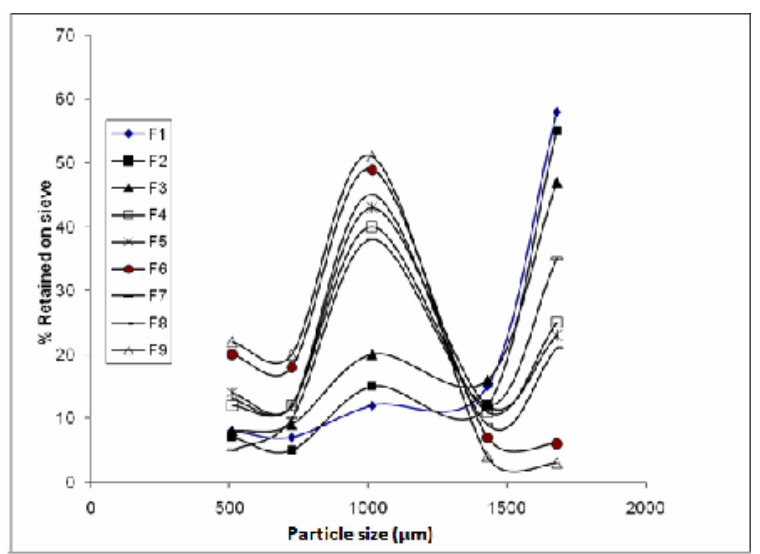

Fig. 4: Particle size distribution curves for IBU-GL pellets of different batches.

The yield in desired range was increased with increase in the amount of GL and the speed of agitation. This may be due to droplet size reduction with shear leading to increase in the yield. GL reduces droplet coalescence causing reduction in particle size. This may be 
attributed to better emulsification at higher GL content. Therefore the yield in the desired size range was increased with increase in amount of GL. These results are in agreement with results of Maheshwari et al. $2003^{8}$ with ibuprofencetyl alcohol pellets.

\section{In-vitro drug release studies}

Only the pellets in the desired size range 800-1200 $\mu \mathrm{m}$ were selected for release studies. The in-vitro drug release of IBU from different pellets in $\mathrm{HCl}$ buffer of $\mathrm{pH} 1.2$ (to mimic the acidic conditions in the stomach) was carried out for $2 \mathrm{hrs}$ as the pellets are not expected to retain longer in the stomach. While the release in phosphate buffer of $\mathrm{pH} 7.4$ (to mimic the environment in the small intestine) was studied for $8 \mathrm{hrs}$. The drug dissolution profiles in $\mathrm{HCl}$ buffer of $\mathrm{pH} 1.2$ for various batches are shown in figure 5 . Very low percentage of drug was released in the acidic medium (7.3 - 9.6\% from different pellets) which is favorable for IBU as its absorption was shown to occur mainly from the intestine and to a lesser extent from the stomach $^{27}$. This is also favorable to avoid its gastric irritation and ulcerogenic effect. IBU with a pka value of 4.45 shows $\mathrm{pH}$-dependent solubility, with a 154 fold higher solubility at $\mathrm{pH} 7$ compared to $\mathrm{pH} 2.2^{28}$, so its dissolution is hindered in acidic media.

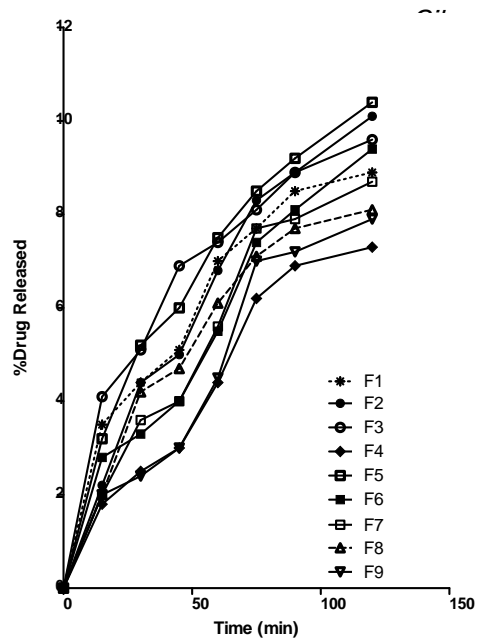

Fig. 5: In-vitro drug release profiles of IBU from different prepared batches in $\mathrm{HCl}$ buffer of $\mathrm{pH}$ 1.2. Each point is the average of 3 experiments.

Dissolution profiles of the drug from different prepared pellets in phosphate buffer of $\mathrm{pH} 7.4$ are shown in figure 6. The release was found to be extended for up to $8 \mathrm{hrs}$, only 77- $90 \%$ drug was released by the end of the release experiment. It has been suggested that, because of the high hydrophobicity of lipid materials in the pellets, the release medium is not able to diffuse through the matrix and can progress in the dosage form by dissolving the grains of drug in contact with it. The dissolution of the drug particles on the surface of the matrix allows the formation of channels, from which the drug is slowly released ${ }^{29}$.
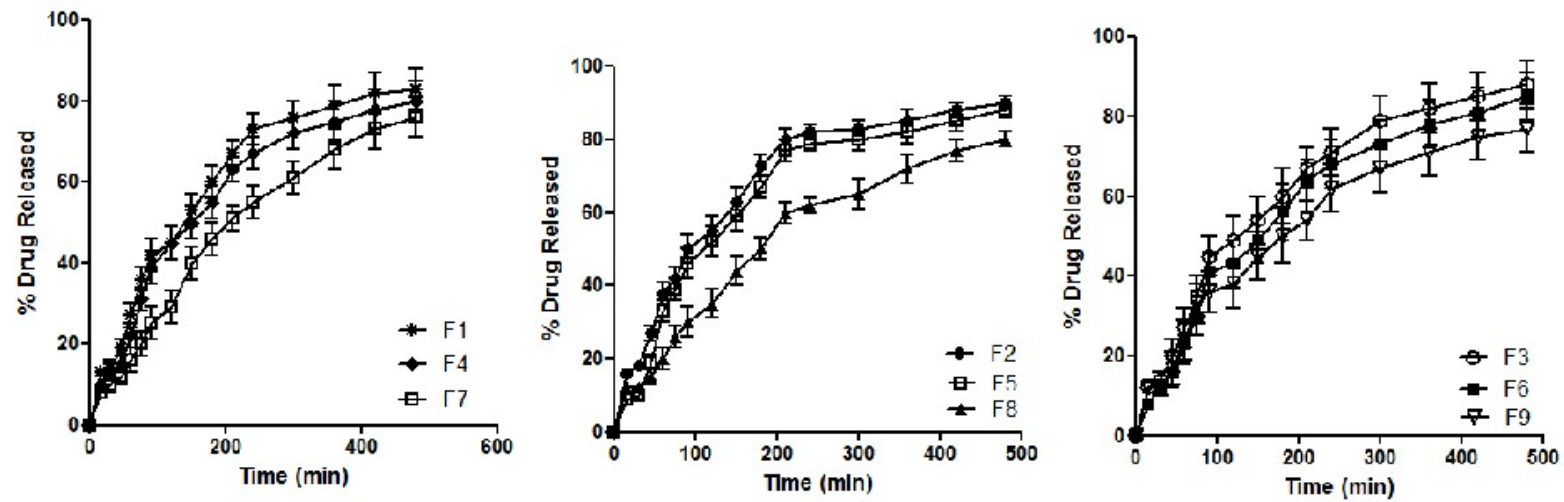

Fig. 6: Effect of amount of GL on the in-vitro release of IBU from different prepared batches in phosphate buffer of $\mathrm{pH}$ 7.4. Each point is the average of 3 experiments. 
As only the pellets in the desired size range $800-1200 \mu \mathrm{m}$ were selected for release studies, the effect of speed of agitation was not seen. The release of IBU was significantly affected by amount of GL (Fig. 6); which could be attributed to the increase of lipid matrix density and in the diffusion path length which the drug molecules have to transverse ${ }^{29}$.

\section{Conclusion}

The IBU-GL pellets prepared by melt solidification technique were spherical with smooth surface. Process yield, drug loading, and entrapment efficiency were high for all batches. Significant release retarding effect for more than 8 hrs. was obtained in $\mathrm{pH}$ 7.4. Increasing amount of GL adversely affected the surface properties, but yield and entrapment efficiency were not adversely affected. Increasing GL amount also retarded the drug release. Gelucire 50/13 can be considered as an effective carrier for the preparation of pellets for sustained delivery of IBU.

\section{REFERENCES}

1- T. Schaefer, P. Holm and H. G. Kristensen, "Melt granulation in a laboratory scale high shear mixer", Drug Dev. Ind. Pharm., 16, 1249 (1990).

2- A. Jonansen, T. Schaefer and H. G. Kristensen, "Evaluation of melts agglomeration properties of polyethylene glycols using a mixertorque rheometer", Int. J. Pharm., 183, 155 (1990).

3- O. L. Sprockel, M. Sen, P. Shivanand and W. Prapaitrakul, "A melt extrusion process for manufacturing matrix drug delivery systems", ibid., 155, 19 (1997).

4- C. De Brabander, C. Vervaet and J. P. Remon, "Development and evaluation of sustained release mini-matrices prepared via hot melt extrusion", J. Control. Release, 89, 235 (2003).

5- C. M. Adeyeye and J. C. Price, "Development and evaluation of sustained-release ibuprofen-wax microspheres. I. Effect of formulation variables on physical characteristics", Pharm. Res., 8, 1377 (1991).

6- C. M. Adeyeye and J. C. Price, "Development and evaluation of sustained release ibuprofen- wax microspheres II-
In-vitro dissolution studies", ibid., 11, 575 (1994).

7- N. Follonier, E. Doelker and E. T. Cole, "Evaluation of hot melt extrusion as a new technique for the development of polymer base pellets for sustained release capsules containing high loadings of freely soluble drugs", Drug Dev. Ind. Pharm., 20, 1323 (1994).

8- M. Maheshwari, A. R. Ketkar, B. Chauhan, V. P. Patil and A. R. Paradkar, "Preparation and characterization of ibuprofen-cetyl alcohol beads by melt solidification technique: effect of variables", Int. J. Pharm., 261, 57 (2003).

9- A. R. Paradkar, M. Maheshwari, A. K. Tyagi, B. Chauhan and S. S. Kadam, "Preparation and characterization of flurbiprofen beads by melt solidification technique", AAPS Pharmscitech., 4, 1 (2003).

10- F. Siepmann, S. Muschert, M. P. Flament, P. Leterme, A. Gayot and J. Siepmann, "Controlled drug release from Gelucirebased matrix pellets: Experiment and theory", Int. J. Pharm., 317, 136 (2006).

11- B. Chauhan, S. Shimpi, K. R. Mahadik and A. Paradkar, "Preparation and evaluation of floating risedronate sodiumGelucire 43/01 formulations", Drug Dev. Ind. Pharm., 31, 851 (2005).

12- W. Bowtle, "Lipid formulations for oral drug delivery", Pharm. Tech. Europe., 12, 20 (2000).

13- A. Ainaoui and J. M. Vergnaud, "Modeling the plasma drug level with oral controlled release forms with lipidic Gelucire", Int. J. Pharm., 169, 155 (1998).

14- A. Ainaoui, E. M. Ouriemchi, D. Bidah, M. K. El Amrani and J. M. Vergnaud, "Process of drug release with oral dosage forms with lipidic gelucire matrix", J. Polym. Eng., 17, 245 (1997).

15- M. T. Sheu, A. Hsia and H. O. Ho, "Polyglycolized saturated glycerides as carrier and enhancer for drug penetration", Chin. Pharm. J., 53, 107 (2001).

16- A. B. Dennis, S. J. Farr, I. W. Kellaway, G. Taylor and R. Davidson R, "In-vivo evaluation of rapid release and sustained release Gelucire capsule formulations", Int. J. Pharm., 65, 85 (1990). 
17- S. A. Barker, S. P. Yap, K. H. Yuen, C. P. McCoy, J. R. Murphy and D. Q. M. Craig, "An investigation into the structure and bioavailability of $\alpha$-tocopherol dispersion in Gelucire 44/14", J. Control. Release, 91, 477 (2003).

18- D. M. Patel, N. M. Patel, V. F. Patel and D. A. Bhatt, "Floating granules of ranitidine hydrochloride-Gelucire 43/01: formulation optimization using factorial design", AAPS Pharm. Sci. Tech., 8 (2), Article 30 (2008).

19- I. Ghebre-Sellassie and A. Knoch, "Pelletization Techniques, Encyclopedia of Pharmaceutical Technology", Marcel Dekker Inc, New York, 1995, p. 369.

20- D. V. Gowda, N. Rajesh, A. Moin, H. G. Shivakumar and Siddaramaiah, "Controlled release behaviour of nifedipine from the pellets of Gelucire/microcrystalline cellulose blends", International Journal of Pharm. Tech. Research, 2, 1215 (2010).

21- J. Hamdani, J. A. Moës and K. Amighi, "Physical and thermal characterisation of Precirol ${ }^{\circledR}$ and Compritol ${ }^{\circledR}$ as lipophilic glycerides used for the preparation of controlled-release matrix pellets", Int. J. Pharm., 260, 47 (2003).

22- A. I. Arida, B. Amoro, M. Jaghbir, M. Elalem, R. Sabri and R. Abuzeid, "Development of sustained-release ibuprofen microspheres using solvent evaporation technique", Arc. der Pharmazie, 332, 405 (1999).
23- P. J. Cox, K. A. Khan, D. L. Munday and J. Sujja-areevath, "Development and evaluation of a multiple-unit oral sustained release dosage form for $\mathrm{S}(+)$ ibuprofen: preparation and release kinetics", Int. J. Pharm., 193, 73 (1999).

24- C. De Brabander, C. Vervaet, L. V. Bortel and J. -P. Remon, "Bioavailability of ibuprofen from hot-melt extruded minimatrices", ibid., 271, 77 (2004).

25- R. Bodmeier, J. Wang and $\mathrm{H}$. Bhagwatwar, "Process and formulation variables in the preparation of wax microparticles by a melt dispersion technique for water-insoluble drugs", J. Microencapsul., 9, 89 (1992).

26- A. R. Paradkar, M. Maheshwar, A. R. Ketkar, B. Chauhan, "Preparation and evaluation of ibuprofen beads by melt solidification technique", Int. J. Pharm., 255, 33 (2003).

27- S. S. Adams, R. G. Bough, E. E. Cliffe, B. Lessel and R. F. N. Mills, "Absorption, distribution and toxicity of ibuprofen", Toxicology and Applied Pharmacology, 1, 310 (1969).

28- J. Hadgraft and C. Valenta, $\mathrm{pH}$, "pKa and dermal delivery", Int. J. Pharm., 200, 243 (2000).

29- S. K. Jain and A. Gupta, "Development of Gelucire 43/01 beads of metformin hydrochloride for floating delivery", AAPS PharmSciTech, 10, 1128 (2009). 